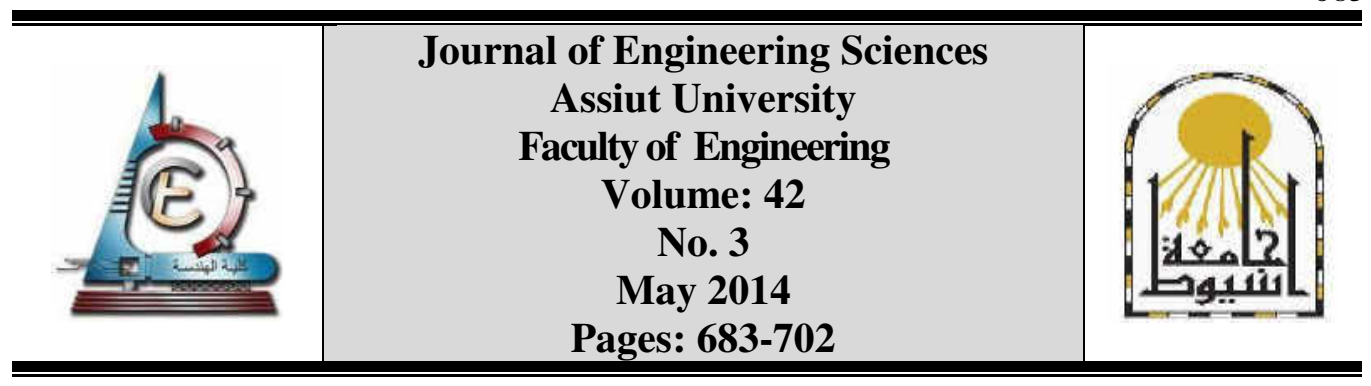

\title{
INVESTIGATION OF CAVITATION DAMAGE PROGRESS IN THE INCUBATION PERIOD USING STEPWISE EROSION AND IMAGE PROCESS TECHNIQUES
}

\author{
M. Osman ${ }^{1}$ and S. M. Ahmed ${ }^{2, *}$ \\ ${ }^{1,2}$ Mechanical Eng. Depat. College of Engineering, Majmaah University, Saudi Arabia
}

Received 20 March 2014; accepted 15 April 2014

\begin{abstract}
Cavitation damage process of stainless steel during the incubation period was detected and evaluated using stepwise erosion technique and image processing. The damage experiments were conducted using the vibratory test rig. The scanning electron microscope images at different locations on eroded surface using stepwise technique were presented. In addition, the surface roughness was measured with the testing time. Discrete wavelet transforms and fractals had been adopted to extract the relevant features. It was found that the feature parameters: Shannon entropy, energy loss, fractal dimension and intercept show a fairly linear increase with the testing time. It was also observed that the feature parameters have reasonable correlations with the roughness parameters.
\end{abstract}

Keywords: Cavitation erosion, electron microscopy, surface analysis, stainless steel

\section{Introduction}

Cavitation is defined as the repeated nucleation, growth, and violent collapse of vapor cavities, or bubbles, in a liquid [1-3]. These collapses exert intense stress pulses, damage and cause material loss and erosion on nearby solid surfaces. Cavitation damage is one of the major problems confronting the designers and users of modern high-speed hydrodynamic systems as reported by Hammitt [1]. It is a serious problem in hydraulic turbines, pumps, valves, control devices, hydraulic structures, sluices, energy dissipators, ship propellers, hydrofoils, bearings, diesel engine wet cylinder liners, aircraft engines, sonar domes, acoustic signal devices, and processing and cleaning equipment [2]. This damage represents a significant problem related to the operating life and economic values of these components [4]. One of the most common methods for predicting the amount of cavitation erosion is based on measurement of mass loss from materials. However, this method has limitations, because the weight loss is global while the cavitation erosion is a local phenomenon. Besides, this method overlooks the early stages of the effects of the

\footnotetext{
* Corresponding author.

E-mail address: shemy2007@yahoo.com
} 
JES, Assiut University, Faculty of Engineering, Vol. 42, No. 3, May 2014, pp. 683 - 702

cavitations and sums only the overall resulting material erosion. However, under cavitation damage, the material surface may be deformed and cracked causing design changes of this surface even before the material begins to have a detectable loss. Therefore, it is essential to characterize the surface change in the early stage of cavitation. The eroded features such as plastic deformation, fracture initiation and its progress are dependent on the load history and surface properties under cavitation erosion. Hence, of particular importance in the recent researches is the need to develop numerical methods providing a reliable and accurate characterization of surface topography with the change in the time.

Literature reports several types of devices and techniques used in laboratory tests for collecting data of cavitation erosion. The ultrasonic vibratory test rigs are the most commonly used to conduct the accelerated tests, allowing a large quantity of data to be accumulated over a relatively short period of time using a wide variety of test conditions, liquids and materials. As reported by Hammitt [1] most of the data obtained from such tests are very helpful in clarifying the cavitation erosion mechanism. A consensus showed that material removal in multiple-impact situations (e.g. cavitation erosion, liquid-droplet erosion, and of solid-particle erosion) is not a result of single impulses or impacts, but rather, it is damage accumulations over thousands of impacts before a particle is dislodged as discussed by Preece and Hansson [2].

It is well established that the rate of erosion is a function of the exposure period, where this rate increases from negligible values, reaches a maximum, then decreases and levels off to a steady value [5]. Thus, cavitation erosion history of a material can be divided into four periods according to ASTM standards G-32 [3]: an incubation period; an acceleration period; a steady-state period and attenuation period. Among these periods, incubation period gained a large share of the research as it represents the importance of the process of cavitation erosion in general due to the existence of plastic deformation, pit formation and surface hardening. The spread of cracks, which control removals often occur in successive periods. Some authors [6-9], in their experimental works found that the pits have a minimal effect in developing erosion. From the analysis of the eroded surface and the dislodged particles for different test conditions, it was found [7, 10-12] that the plastic deformation development and the surface hardening lead to the fatigue failure. However, there is little research [13] that tries to evaluate the erosion resistance of metallic materials by analyzing the plastic deformation behavior of metallic materials during the incubation period. As the cavitation erosion is locally distributed, the surface profile technique may not be a representative to the whole damage on the surface.

Recently, the image analysis technique is considered a worthwhile tool for being able to perform analyzes of fast, inexpensive and non-destructive for many processes. Image analysis is efficient in many tribological applications, e.g. wear debris morphology, texture classification and surface quality in machining as well as it is an appropriate tool to qualitatively and quantitatively characterize the early stage of cavitation erosion process $[11,12$, and 14]. The topographical images of cavitation erosion surfaces at water and oilin-water emulsions were quantified using fractal and wavelet techniques $[15,16]$. The image feature parameters of both techniques and the weight loss decrease with adding oil to water. Also, it was found that the variation of image feature parameters versus concentration of oil-in-water emulsions had a general trend that did not depend on magnification factor. 
M. Osman and S. M. Ahmed, Investigation of cavitation damage progress in .............

In the present work, fractal and wavelet analysis of the stepwise erosion combined with relocation scanning electron microscopy were used to delineate the real erosion processes within the incubation period in considerable details. The surface roughness evolution within the incubation period was also measured for the stepwise erosion.

\section{Image analysis methods}

Various image processing tools can be used to analyze damage surface images and extract the relevant features. Wavelet transforms and fractals were adopted in this study, and are described briefly below and elsewhere $[15,16]$.

\subsection{Wavelet and wavelet packet decomposition}

The main advantage of using wavelets is that it provides multi-resolution analysis to improve the image quality obtained from microscopy techniques, such as SEM and others. The different wavelet transform functions filter out different range of frequencies (i.e. sub bands) by decomposing the image into low frequency and high frequency sub band images. When applying the wavelet transform, original image can be decomposed into four sub-band images. The decomposition process is recursively applied to the low-frequency sub-band to generate the next level of the hierarchy. That is, the two-dimensional discrete wavelet transform (2D DWT) [17] decomposes an image into several sub-bands according to a recursive process as shown in Fig 1. These include LH1, HL1 and HH1 which represent detail images and LL1 which corresponds to an approximation image, which is then decomposed into second-level approximation and detail images. This process is repeated to achieve the desired level of resolution. The obtained coefficients for the approximation and detailed sub-band images are useful features for texture categorization.

\begin{tabular}{|c|c|c|c|}
\hline $\mathbf{L L}_{3}$ & $\mathrm{HL}_{3}$ & \multirow{2}{*}{$\mathrm{HL}_{2}$} & \\
\cline { 1 - 1 } $\mathbf{L H}_{3}$ & $\mathrm{HH}_{3}$ & \\
\cline { 1 - 2 } $\mathbf{L H}_{2}$ & \multirow{2}{*}{$\mathbf{H L}_{2}$} & \\
\hline & & \\
& & \\
& & \\
& & \\
& & \\
& & \\
\end{tabular}

Fig. 1. Pass-band structure for a two dimensional sub-band transform with three levels.

As an extension of the standard wavelets, wavelet packets represent a generalization of the multi-resolution analysis and use the entire family of sub-band decompositions to generate an over complete representation of images [18]. There are many outstanding properties of wavelet packets that encourage research in many widespread fields.

An image is decomposed into one approximation and three detail images in 2-D discrete wavelet packet transforms (2-D DWPT). The approximation and the detail images are then decomposed into a second-level approximation and detail images, and the process is repeated. The wavelet packet tree for 3-level decomposition is shown in Fig. 2. The standard 2-D DWPT can be implemented with a low-pass filter $\mathrm{h}$ and a high-pass filter $\mathrm{g}$ [19]. The 2-D DWPT of an $\mathrm{N} \times \mathrm{M}$ discrete image $\mathrm{A}$ up to 
level $\left(P+1 \quad\left(P \min \left(\log _{2} N+\log _{2} M\right)\right)\right.$, is recursively defined in terms of the coefficients at level $\mathrm{p}$ as follows:

$$
\begin{gathered}
c_{4 K,(i, j)}^{P+1}=\sum_{m} \sum_{n} h(m) h(n) c_{K,(m+2 i, n+2 j)}^{P} \\
c_{4 K+1,(i, j)}^{P+1}=\sum_{m} \sum_{n} h(m) g(n) c_{K,(m+2 i, n+2 j)}^{P} \\
c_{4 K+2,(i, j)}^{P+1}=\sum_{m} \sum_{n} g(m) h(n) c_{K,(m+2 i, n+2 j)}^{P} \\
c_{4 K+3,(i, j)}^{P+1}=\sum_{m} \sum_{n} g(m) g(n) c_{K,(m+2 i, n+2 j)}^{P}
\end{gathered}
$$

Where $c_{0}^{0}$ is image $\mathrm{A}$ and $\mathrm{K}$ is an index of the nodes in the wavelet packet tree denoting each sub-band; $h$ and $g$ are the filter coefficients of low-pass and high-pass filters, respectively. Supposing that Haar basis has been used, $\mathrm{h}=\{-0: 7071 ; 0: 7071\}$, and $\mathrm{g}=$ $\{0: 7071 ; 0: 7071\}$. At each step, the image $c_{K}^{P}$ is decomposed into four quarter-size images $c_{4 K}^{P+1}, c_{4 K+1}^{P+1}, c_{4 K+2}^{P+1}, c_{4 K+3}^{P+1}$. The capital letters ( $\mathrm{N}$ or $\left.\mathrm{M}\right)$ are maximum constants defined by the image size. However, small letters ( $\mathrm{m}$ or $\mathrm{n}$ ) are defined at each step. For example, when $\mathrm{P}=5, \mathrm{P}$ can be $1,2,3$, or 4 and so on.

The Shannon entropy in different sub-bands is computed from the sub-band coefficient matrix as:

$$
\begin{gathered}
\operatorname{Entropy~}_{P}(k)=-\sum_{i} \sum_{j}\left|c_{k,(i, j)}^{P}\right|^{2} \log \left|c_{k,(i, j)}^{P}\right|^{2} \\
\operatorname{Energy}_{P}(k)=-\sum_{i} \sum_{j}\left|c_{k,(i, j)}^{P}\right|^{2}
\end{gathered}
$$

Where Energy $\mathrm{p}^{(\mathrm{k})}$ and Entropy $\mathrm{p}^{(\mathrm{k})}$ are the energy and entropy of the image projected onto the subspace at node $(\mathrm{p}, \mathrm{k})$. The entropy of each sub-band provides a measure of the image characteristics in that sub-band. The energy of each sub-band provides a measure of the image characteristics in that sub-band. The energy distribution has important discriminatory properties for images and as such can be used as a feature for texture classification. From the equations above, it follows that the wavelet entropy is minimum when the image represents an ordered activity characterized by a narrow frequency distribution, whereas the entropy is high when an image contains a broad spectrum of frequency distribution.

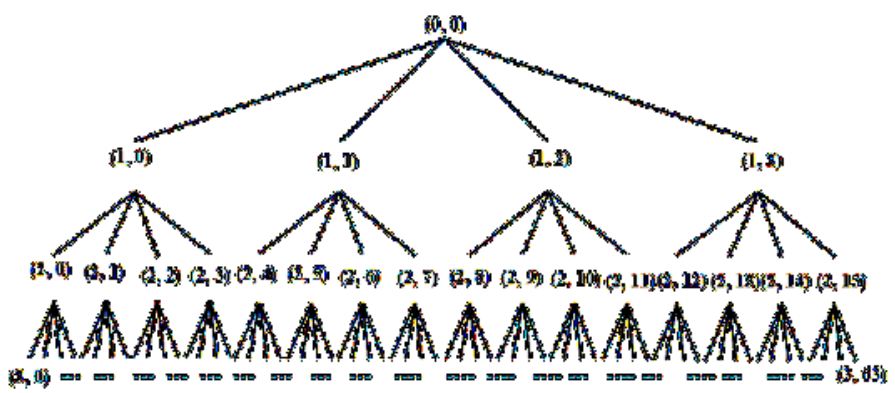

Fig. 2. Wavelet packet tree decomposition. 
M. Osman and S. M. Ahmed, Investigation of cavitation damage progress in .............

\subsection{Fractal analysis}

Fractal geometry is a well-known non-traditional method which has found many applications in science and engineering. It is common knowledge that many objects in nature are of irregular form which cannot be described by Euclidean geometry. These nonEuclidean objects are called fractals, and can be described by non-integer numbers. These non-integer numbers define the fractal dimension (FD) of an object. The main concept of fractal geometry analysis is that a fractal dimension can be considered as a quantitative measure of object surface heterogeneity because of its inherent self-similarity features. In a simplified representation, one could interpret the fractal dimension as a measure of heterogeneity of a set of points on a plane, or in space. Many works were reported on the application of fractal geometry to describe complex physical phenomena such as turbulence, brittle fracture of materials, machining, and tool wear [20, 21]. Fractal characterizations have also been used to describe complex two or three-dimensional surfaces, such as deposited surfaces [22], wear-erosion surfaces [23, 24], and wear particles [25-28]. Eroded surfaces are likely to be multi-fractal because they are produced by the accumulative effects of multiple impacts, i.e., rough surfaces. Fractal dimension at low frequency describes raw structures, while fractal dimension at high frequency represents texture. Textural fractal should define very fine feature in object boundary, surface, area, or volume. Structural fractal should describe an overall object morphology. Therefore the distinction between structural and textural fractals allows investigators to characterize microstructure and macrostructure effects on the surface behavior.

Various fractal analytical techniques are applied to micrograph images of eroded surfaces such as Fourier, Kolmogorov, Korcak, Minkowski, root mean square, Slit Island, etc. These techniques differ in computational efficiency, numerical precision and estimation boundary. The most efficient procedure for measurement of the fractal dimension FD of eroded surfaces, and one which allows characterization of anisotropic surface as well, seems to be through Fourier analysis [29, 30]. Therefore, Fourier analysis is adopted to estimate fractal values in this work. For a surface image represented by the function $\mathrm{I}(\mathrm{x}, \mathrm{y})$, the power spectral density (PSD) is equal to the square of the Fourier transformation $\mathrm{F}(\mathrm{u}, \mathrm{v})$ of the surface function $\mathrm{I}(\mathrm{x}, \mathrm{y})$. The power spectral density function is defined as;

$$
S(u, v)=|F(u, v)|^{2}
$$

Where $\mathrm{u}$ and $\mathrm{v}$ are the spatial frequencies (number of waves per unit wave length) in the $\mathrm{x}$ and $\mathrm{y}$ directions respectively. The PSD is converted to the polar coordinate system $\mathrm{S}$ (f), such that $f=\sqrt{u^{2}+v^{2}}$. The value of $\mathrm{S}(\mathrm{f})$, at each radial frequency $\mathrm{f}$, is averaged over angular distributions. For a fractal surface, Fig.3 (a), the power spectrum shows a linear variation between the logarithm of $S(f)$ and the logarithm of the frequency as shown in Fig.3 (b).The slope of the linear regression line $\beta$ is related to FD by equation $[13,31]$ :

$$
F D=\frac{8-\beta}{2}
$$

It is reported in literature that fractal dimension and intercept are significant fractal parameters that describe the irregularity and complexity of the surfaces. Moreover, the 
intercept correlates well with the overall magnitude of roughness of the observed texture appearance of the surface images. In the present study, fractal analysis is going to be used to assign numerical values to indicate the development of stepwise erosion for cavitation eroded surfaces.
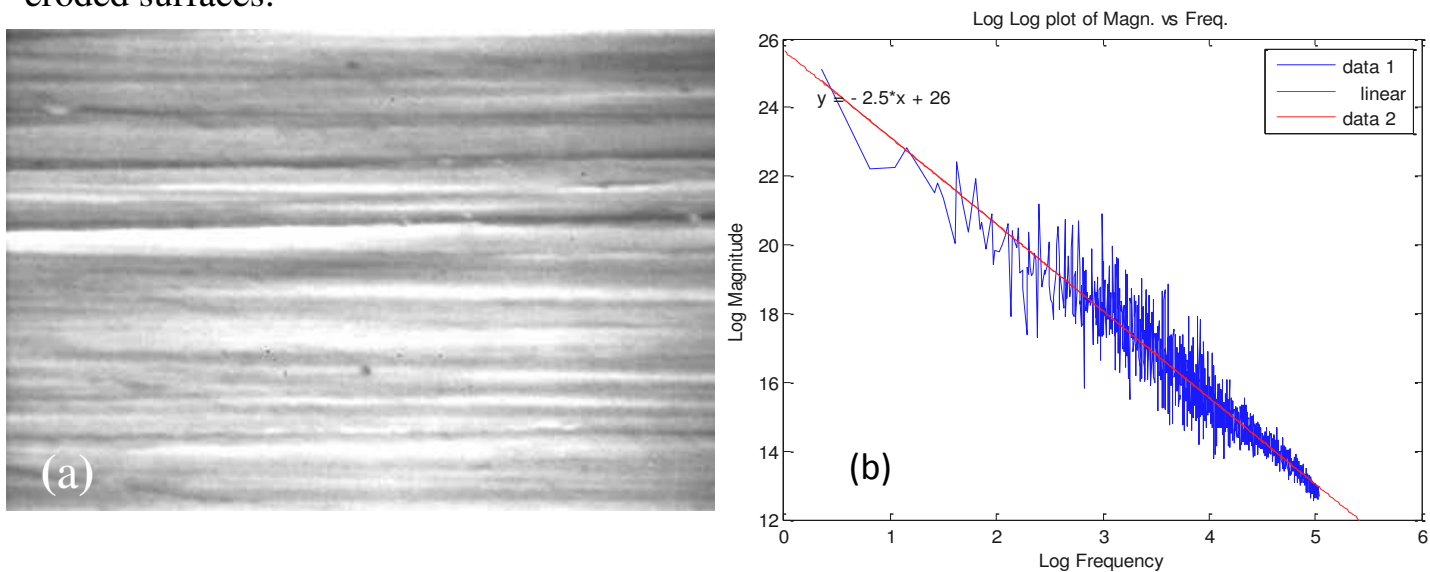

Fig. 3. (a) A surface image of polished surface, and (b) Power spectrum of its Fourier transform, plotted as directionally averaged of log (PSD) versus log (frequency).

\section{Experimental procedure}

The test material was austenitic stainless steel SUS 304, whose nominal mechanical properties and chemical composition are shown in Table 1. To ensure metallurgical uniformity, all test specimens were cut from single round rod of the material. The round specimens were machined to flat-surfaced discs of diameter of $15.9 \mathrm{~mm}$ and a height of 11 $\mathrm{mm}$ with threaded sections for attachment to the cavitation vibratory horn. . Initial surface roughness of the specimens is known to be of a considerable importance in creating pits [7], to facilitate crack initiation [32], to develop the fracture and to increase the rate of linear particle dislodgment [33]. To minimize these problems in the present study, the working faces of specimens were highly polished with grade 3000 dry emery paper.

Cavitation erosion experiments were performed using ultrasonic cavitation test apparatus shown in Fig. 4, which is in accordance with ASTM standard G32-06 [3]. The apparatus consists of a quartz transducer oscillating at a resonant frequency of $20 \mathrm{kHz}$, attached to an exponentially shaped prop, which amplifies the amplitude of vibration. The double amplitude (peak to peak) was $50 \mu \mathrm{m}$. This alternating sound field generates pressure fluctuation with the horn-tip immersed in liquid. During one half of each vibration cycle, a low pressure is created at the test specimen surface, producing cavitation bubbles. During the other half of the cycle, bubbles collapse at the specimen surface.

The specimens were immersed in $1000 \mathrm{ml}$ open glass beaker, containing $700 \mathrm{ml}$ of tap water. Since the test liquid temperature markedly affects the degree of erosion [34,35], the test water temperature in the beaker was controlled by cooling water circulation to be within $22 \pm 1{ }^{\circ} \mathrm{C}$, as shown in Fig.4.

Four specimens were tested at successive intervals within incubation period, two specimens for capturing the eroded surface image and the other two for surface roughness measurement. Such short experimental time (i.e.: elapsed time is within 5 min.) was 
scheduled to reduce other damage effect on the surface, such as corrosion. The surface roughness was 2-dimensionally measured by a stylus profilometer in the direction of lay and in perpendicular direction to the lay. Then the average value of the surface roughness parameter was estimated for the two directions. The average value of roughness parameters $\mathrm{R}_{\mathrm{a}}$ (mean surface roughness) and $\mathrm{R}_{\mathrm{q}}$ (Root-mean-square roughness) was calculated from five random profiles of sampling length $2.5 \mathrm{~mm}$ at each test time. The duration of each interval time must be sufficient to produce gradually change in the features of the damage. Therefore, a $5 \mathrm{~min}$. was decided for a total of $45 \mathrm{~min}$. of testing on each specimen. The specimens were ultrasonically cleaned in acetone, dried in open air, and weighed by a digital balance with a sensitivity of $0.01 \mathrm{mg}$.

It is known that cavitation erosion is localized and unevenly distributed. So in order for the stepwise erosion results to be representative, photographs were taken at four locations on the surface of the sample, with two near the center and the other two near the edge. For comparative examination, capturing an image of the same area on the surface of the sample after each test requires relocation of the sample in the microscope, which has been done through the rotation of the sample and observing the lay of specimen using small magnification and comparing it to the previous images.

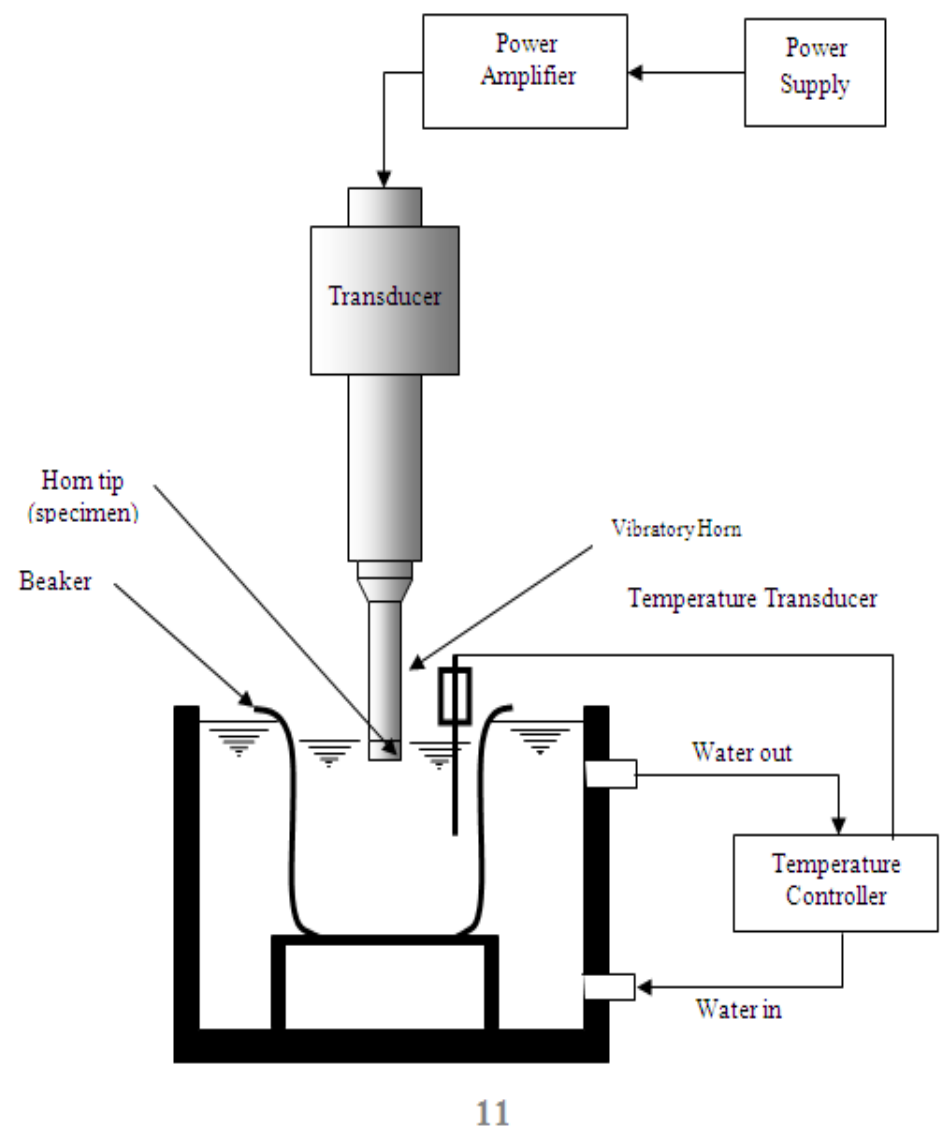

Fig. 4. Schematic view of test apparatus. 
JES, Assiut University, Faculty of Engineering, Vol. 42, No. 3, May 2014, pp. $683-702$

Table 1.

Mechanical properties and chemical composition of SUS 304

\begin{tabular}{|l|l|}
\hline Density & $8027 \mathrm{~kg} / \mathrm{m}^{3}$ \\
\hline Yield stress & $517 \mathrm{MPa}$ \\
\hline Tensile strength & $758 \mathrm{MPa}$ \\
\hline Elastic modulus & $193 \times 10^{3} \mathrm{MPa}$ \\
\hline Hardness, BHN & 240 \\
\hline Elongation percent & 60 \\
\hline $\begin{array}{l}\text { Nominal chemical } \\
\text { composition } \quad \mathrm{C} 0.08 \text { max, Mn 2max, Si 1 max, P 0.045max } \\
\text { S } 0.03 \text { max, Cr 18-20, Ni 8-12 }\end{array}$ \\
\hline
\end{tabular}

\section{Results and Discussion}

\subsection{Morphologies of eroded surfaces}

Figure 5 shows the sequence of images before and after testing for times of 5, $10 \ldots$ and $35 \mathrm{~min}$. It is clearly seen on the images of the edge and center positions at $\mathrm{t}=0 \mathrm{~min}$., there are a number of horizontal parallel polishing lines. A number of black spots seen on the edge images, at $t=5$ min., represents contaminations by dust particles that do not appear on the subsequent images. Therefore, the lines and the spots are not related to the cavitation. It was reported $[6,7]$ that when specimens of ductile austenitic stainless steel were exposed to cavitation, local plastic deformation of its surface was observed. This deformation was in the form of surface undulations with the appearance of defects, such as grain boundaries and slip bands as well as the formation of pits. With the development of testing time, these undulation changes in width and height, but generally they do not change in shape and number. It is noted in these images for plastic deformation slip bands are at least of $40 \mu \mathrm{m}$ in length.. Such long undulations reveal that the shock pressure developed by the bubbles collapse plays a vital role in developing the erosion [9]. The images also show that the loss of significant particles in the evolution of the erosion process began along the slip bands as it is clear from the images of the eroded surface at the edge of time of 20 to $25 \mathrm{~min}$. The particles were fallen off due to fatigue. Once the work hardening capacity of the surface reaches a critical level, fatigue cracks initiate plastically in the slip bands at microstructural discontinuities. The latter, being inhomogeneous and "condensed" plastic strains, changes locally and unevenly the microgeometry of the sample surface (micronotches), also causes local stress concentrations.

Another feature of eroded surface is the pit formation encircled in the images of Fig. 5. The size of these pits is in the order of $\mu \mathrm{m}$. Such a tiny pit arises from the exposure to highly erosive microjets of pressures larger than the strength of the tested material. It is clearly seen from the sequence of images that the shape and diameter of pits do not change at all. Based on this observation on the pits feature and elsewhere [5], it can be concluded that the pits scarcely play an important role in the development of erosion. 
M. Osman and S. M. Ahmed, Investigation of cavitation damage progress in .............
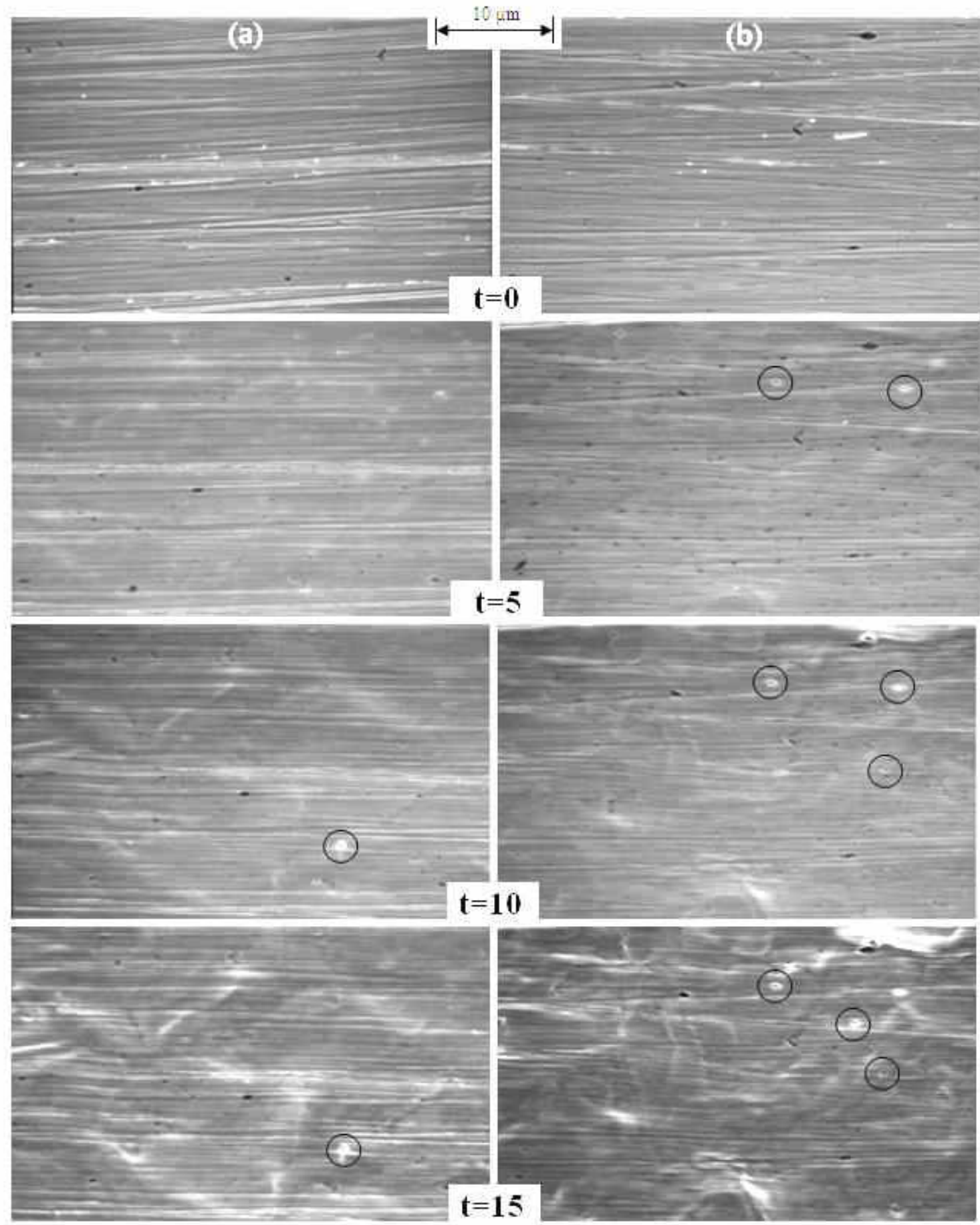

Fig. 5. Sequences of images show the progression of damage with time at two positions on the sample: (a) the center, (b) the edge. 

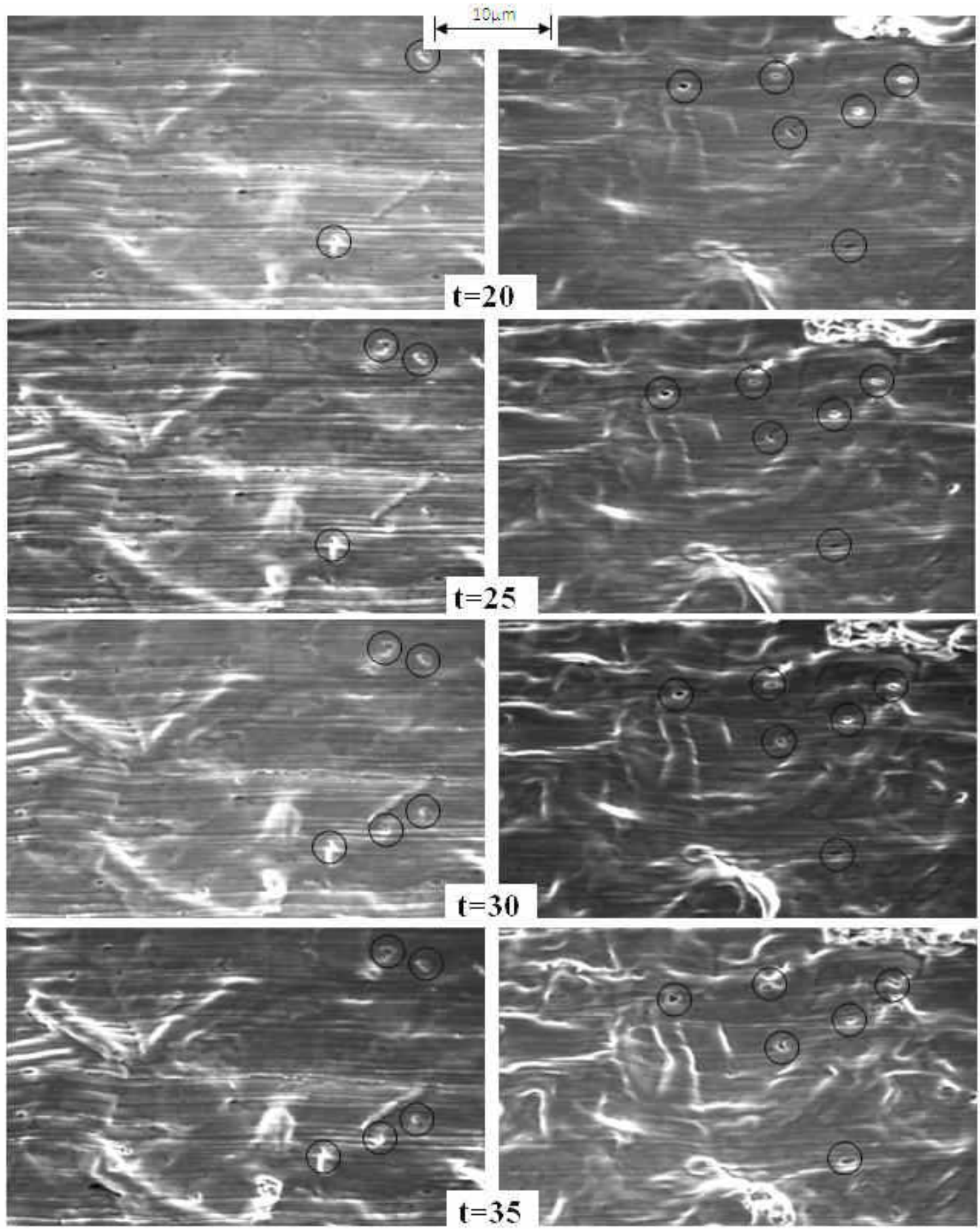

Fig. 5. Continue 
M. Osman and S. M. Ahmed, Investigation of cavitation damage progress in .............

\subsection{Change in surface roughness}

The results of the average roughness $R_{a}$ and the root mean square roughness $R_{q}$ with the test time are plotted in Fig. 6. These data can be divided into two stages based on the incubation period: stage I for $\mathrm{t} \leq 15 \mathrm{~min}$, and stage II for $\mathrm{t}>15 \mathrm{~min}$. In both stages, the roughness values increase linearly with the time, where this rate of the increase is larger in the second stage. In both stages I and II, the material surface is plastically deformed leading to a uniform rate of roughness. Meanwhile, the increase in the rate of roughness in the second stage II is caused by the initiation of cracks as shown at the edge of the image for 20 min. Many results about the measurement of roughness in the incubation stage and subsequent stages were reported in the literature [7, 12, 36]. However, the present measurements show slightly different results compared to the reported results due to the fact that they are the average value of the measurement of two profiles, one in the direction of lay and the other in perpendicular direction to it. The assigned profile includes the whole eroded surface. Similar results have been reported by Yabuki et al. [37] but with different scale for the roughness measurement. They proposed "surface increment percentage" index to represent the surface modification during the incubation period. The increment of the surface area was determined from the material surface profile data, measured through roughness meter. According to this index, the incubation period of cavitation erosion is to be divided into two stages. They found that, in the first stage, the rate of surface increment is related to the hardness of the metallic materials while in the second stage this rate is related to the strain hardening exponent. Therefore, Yabuki et al. [37] concluded that surface increment index can be applied to the rapid determination of the level of erosion resistance, which in turn can lead to the rapid prediction of the service life of material. Recently, Matsmura [38], in his analysis for this methodology, saw that this method is inevitable for the determination of cavitation intensity in hydraulic machines.

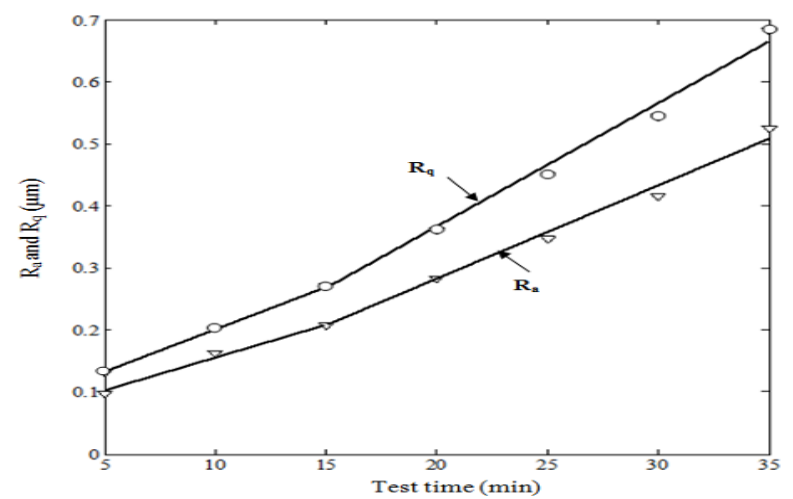

Fig. 6. Change of average $R_{a}$ and RMS roughness $R_{q}$ with test time t.

\subsection{Feature extraction}

In this section, the features parameters of images such as wavelet entropy, wavelet energy, and fractal dimension and intercept values are extracted. Then, these parameters are correlated to the surface roughness parameters. A set of 8 images (4 images per sample at different locations) were collected to represent the whole surface damage. Before any data processing was made, these raw images were visually assessed to avoid any effect on 
JES, Assiut University, Faculty of Engineering, Vol. 42, No. 3, May 2014, pp. 683 - 702

the parameters of image like change in dimension, change in structure value due to contaminations. Therefore the data at test time of $5 \mathrm{~min}$ is omitted due to contamination as reported in Sec. 4.1.

The results of the feature extraction, performed by using 2D-DWPT and fractal analysis described in Sec.2 are given in Figs 7-10. Shannon entropies and energy of wavelet packet decomposition of image were calculated with the testing time from all the selected sub-bands of the image as shown in Figs. 7 and 8. Figures 9 and 10 show the fractal dimensions and intercept for the image of eroded surface at different times. Fitting results between the specimen's image parameters and exposure time and the correlation coefficient, $\mathrm{R}^{2}$, are shown in these figures. Entropy has the best linear correlation with time with adjusted $\mathrm{R}^{2}$ value of 0.98 . Then, followed by intercept, fractal dimension and energy with $R^{2}$ values of $0.90,0.90$, and 0.89 respectively. From these figures, it is shown that the slope of the line of entropy and its intercept increase with time, while it decreases for energy. Tao et al. [39] used the image energy loss D as a characteristic to estimate the corrosion loss. The image loss $\mathrm{D}$ was calculated as follow:

$$
D_{j}=E_{0}-E_{j}
$$

Where $j$ means the exposure time and $E_{0}$ is the energy of uneroded specimen. The energy loss $D_{j}$ versus time is plotted in Fig. 11.

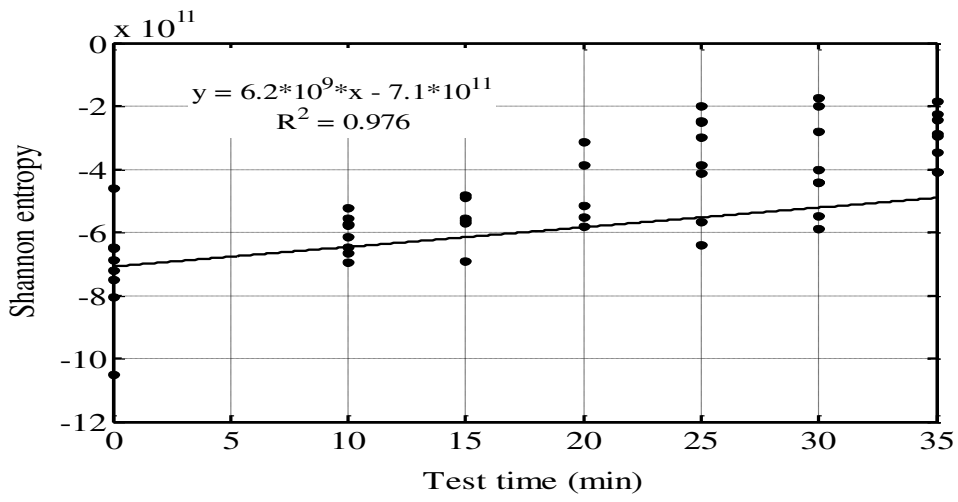

Fig. 7. Change of Shannon entropy with test time t.

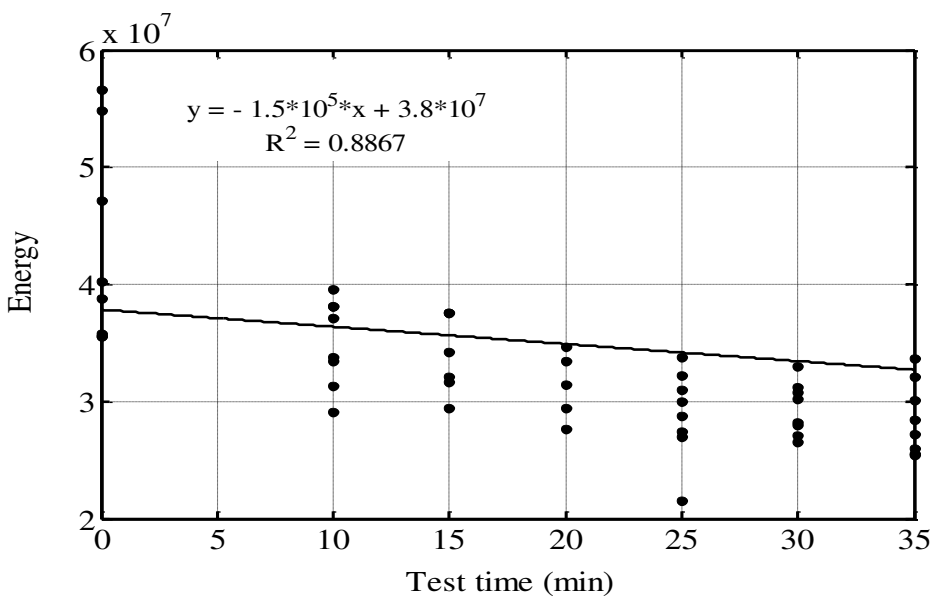

Fig. 8. Change of wavelet energy with test time t. 


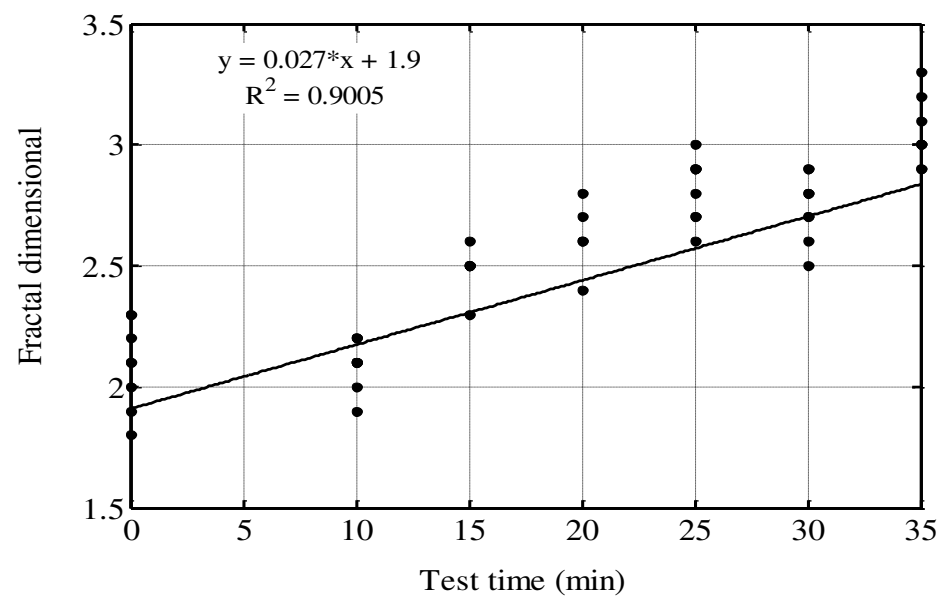

Fig. 9. Change of fractal dimension with test time t.

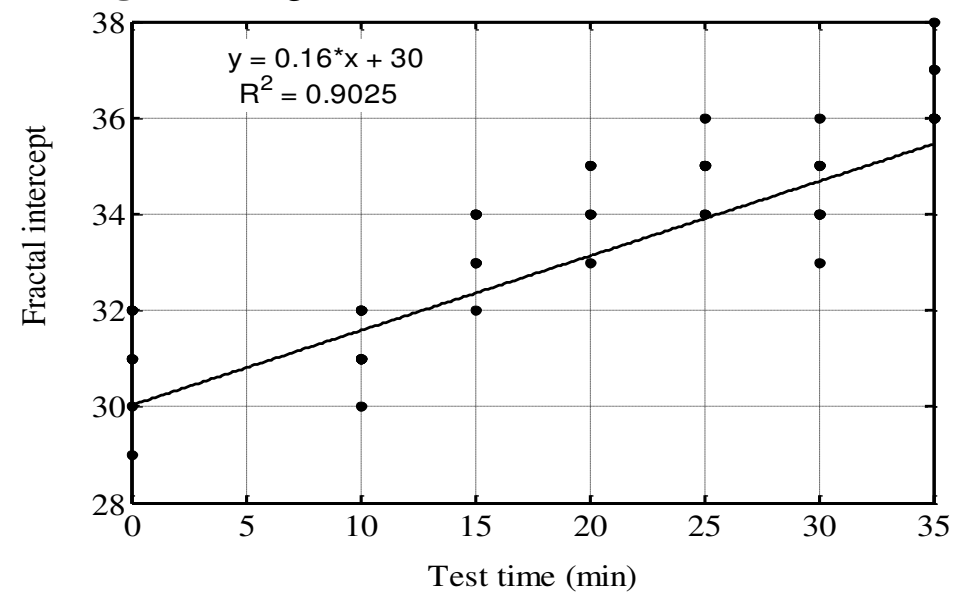

Fig. 10. Change of fractal intercept with test time $t$.

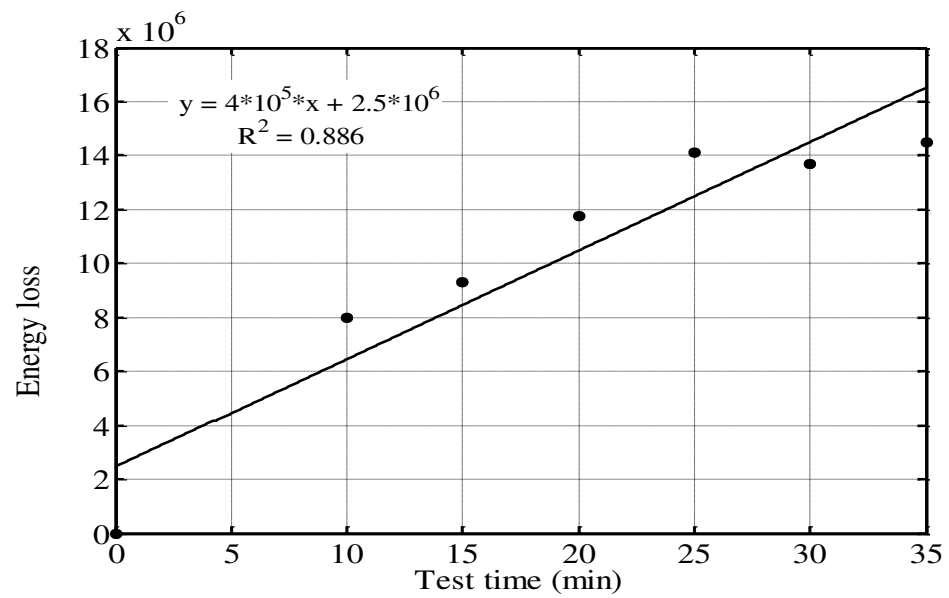

Fig. 11. Change of wavelet energy loss of eroded surface image with the time. 
JES, Assiut University, Faculty of Engineering, Vol. 42, No. 3, May 2014, pp. 683 - 702

From this figure, it can be seen that the energy loss increases with time in a similar manner to the behaviors of entropy, fractal dimension and intercept with time.

Each feature corresponds to a visually recognizable property of the image described in Section 4.1. That is, for uneroded surfaces with homogeneous textures the results show relatively low values of entropy, energy loss, fractal dimension and slope. While, these extracted features have high values for eroded surfaces due to the coarseness of the surface textures. To verify these results, Figs. 12 \& Fig. 13 examines the possible correlation between feature parameters extracted from images and corresponding average roughness, $R_{a}$ and root mean square roughness $R_{q}$, given in Fig.6. It is clear that the correlations of feature parameters with the roughness parameters are similar to the correlations with the testing time. It is worth noting that Shannon entropy exhibits the best correlation with $R_{a}$ and $R_{q}$ with correlation coefficient 0.98 . It can be concluded that the image texture analysis by discrete wavelet analysis and fractal analysis of the cavitation eroded surface can be used to predict the nature variation of cavitation damage.

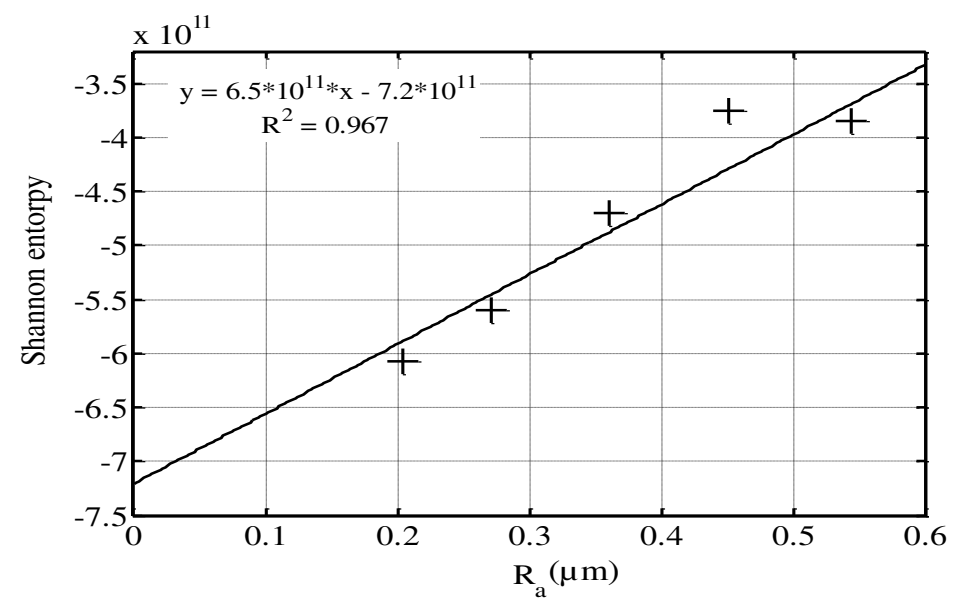

(a)

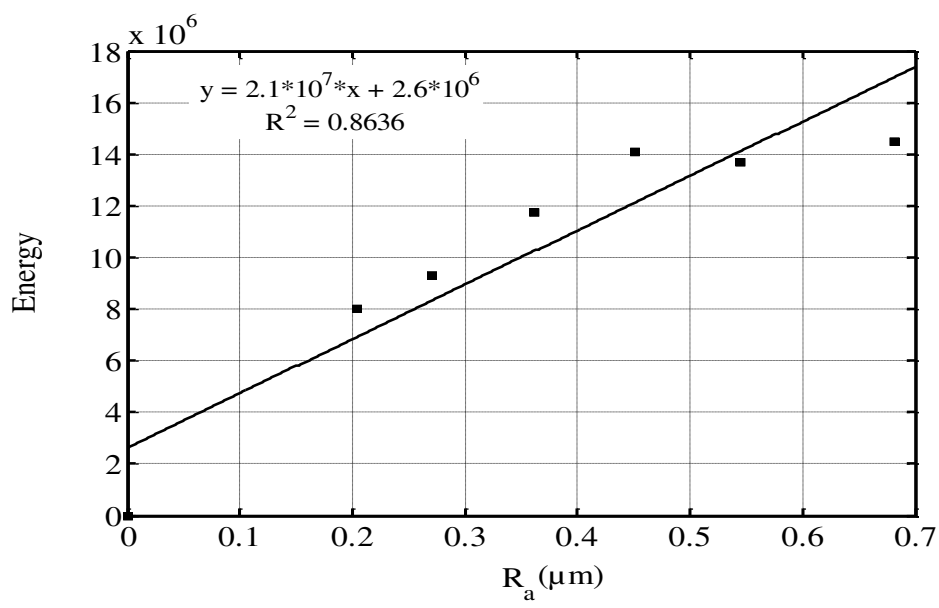

(b) 


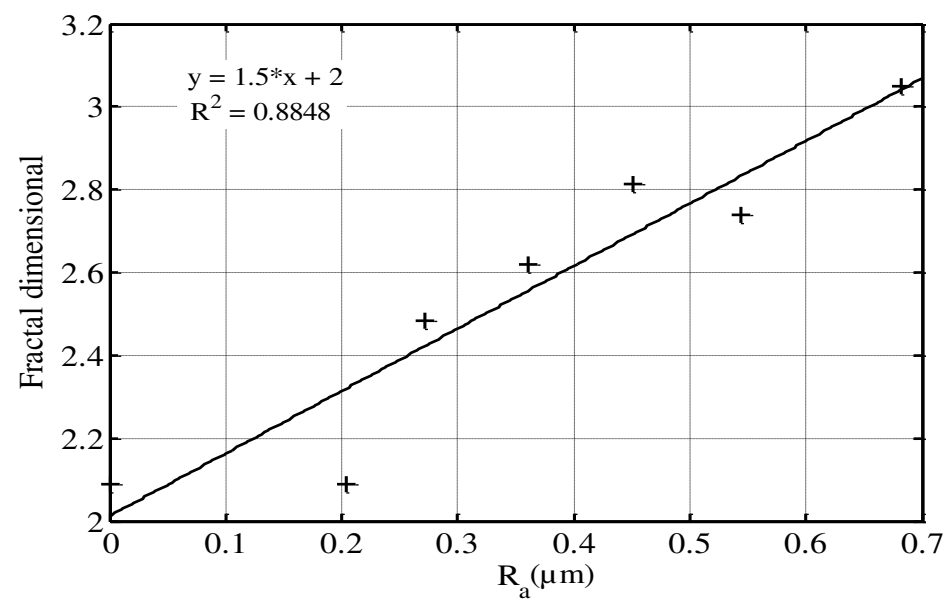

(c)

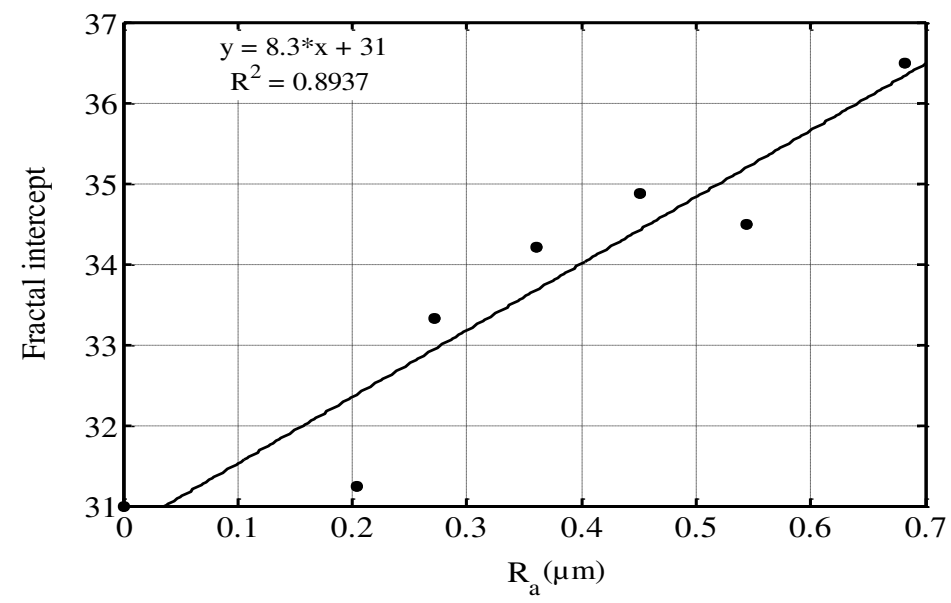

(d)

Fig. 12. Feature parameters: Shannon entropy (a), energy (b), fractal dimension (c), fractal intercept (d) versus $R_{a}$.

\section{Conclusions}

Image processing based on discrete wavelet transform and fractal analysis was used to characterize the cavitation damage images. Those images were captured using the stepwise erosion technique. The following results can be drawn:

(1) The extracted feature parameters; Shannon entropy, energy loss, fractal dimension and fractal intercept change linearly with exposure time.

(2) The relationships between feature parameters and roughness parameters were linear. The best correlation was for Shannon entropy.

(3) The results indicate that the image analysis procedures rep promising techniques since they are effective in characterizing the changes of surface topography with exposure time. 
(4) The surface topography with test time showed the plastic deformation development that leads to the fatigue failure was the predominant mechanism.

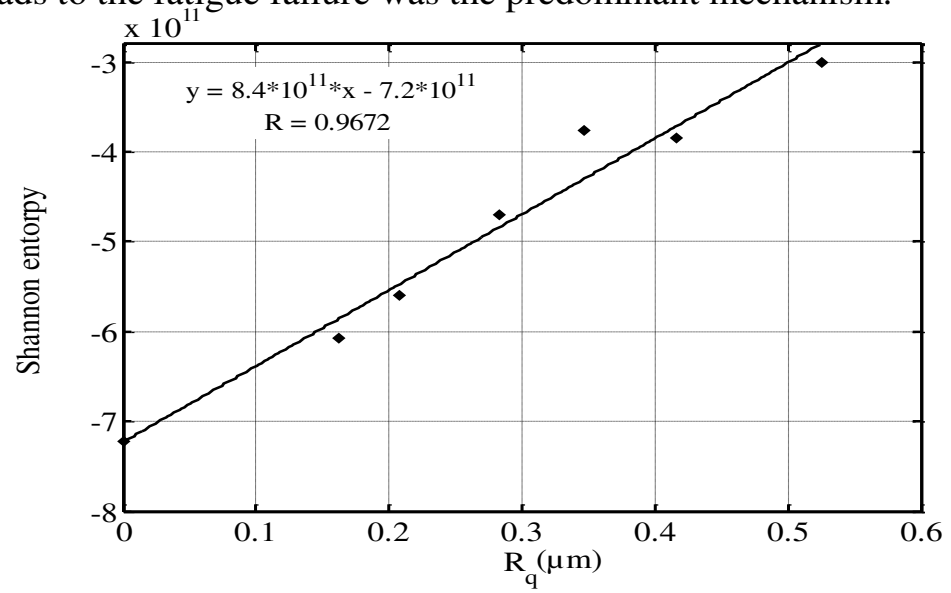

(a)

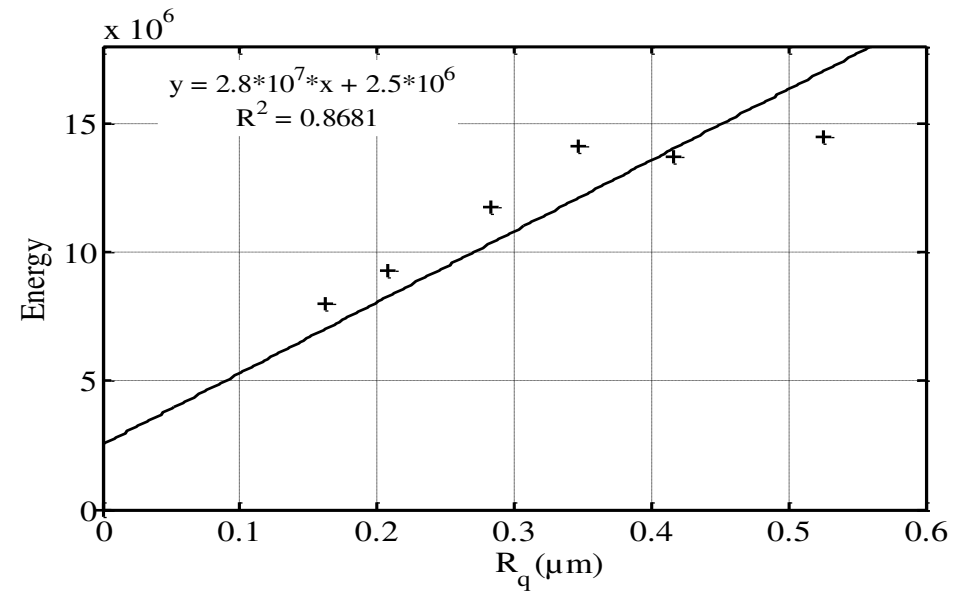

(b)

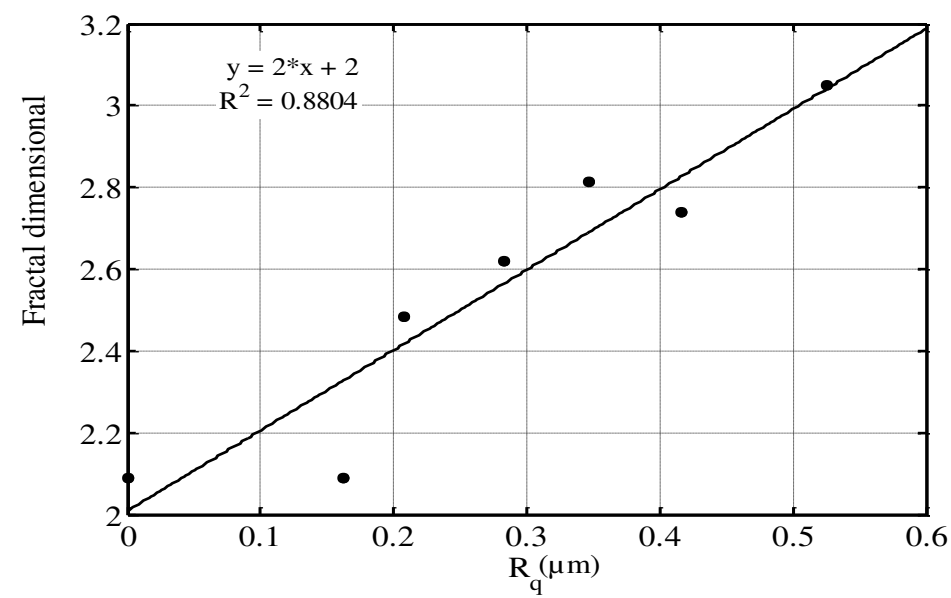

(c) 


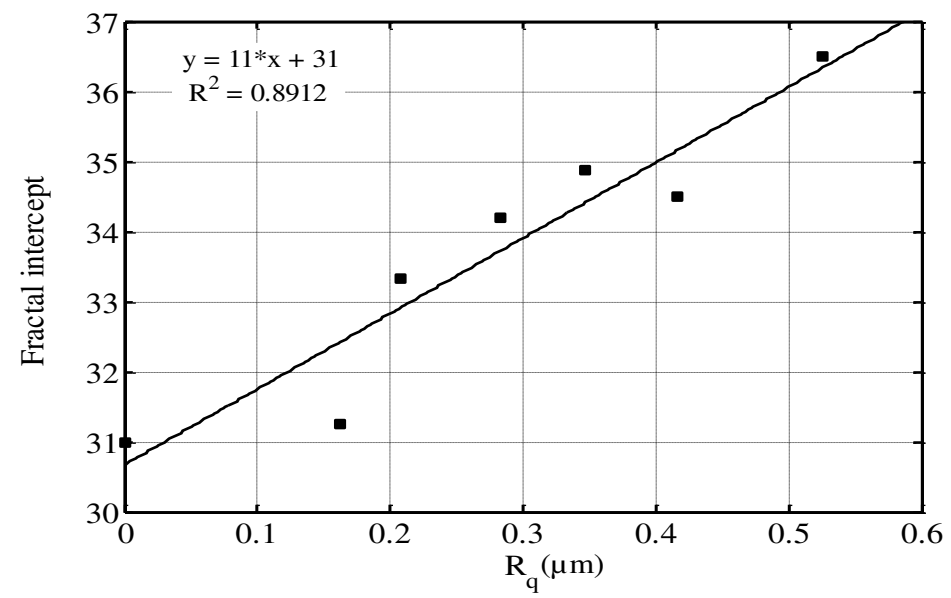

(d)

Fig. 13. Feature parameters: Shannon entropy (a), energy loss (b), fractal dimension (c), fractal intercept (d) versus $R_{q}$.

\section{REFERENCES}

[1] Hammitt FG. Cavitation and multiphase flow phenomena. New York: McGraw- Hill; 1980.

[2] Preece CM, Hanssson ILH. A metallurgical approach to cavitation erosion, Advances in the Mechanics and Physics of Surfaces. Latanision, R. M. and Courtel, R. J., Ed., Harwood Academic 1; 1981, p.199-253.

[3] ASTM Stand-G32-06. Standard test method for cavitation erosion using vibratory apparatus. In: Annual book of ASTM standards, Part 03.02.

Philadelphia, PA: ASTM; 2006. p. 98-112.

[4] March P, Hubble J. Evaluation of relative cavitation erosion rates for base materials, weld overlays, and coatings, Tennessee Valley Authority Engineering Laboratory, Norris, TN, Report No. WR28-1-900-282, September 1996.

[5] ITTC. The Specialist Committee on Cavitation Erosion on Propellers and Appendages on High Powered/High Speed Ships: Final Report and Recommendations to the 24th ITTC. In: Proc. 24th International Towing Tank Conference, 2005; 2:509-542.

[6] Abouel-Kasem A, Ezz El-Deen A, Emara KM, Ahmed SM. Investigation into cavitation erosion pits. ASME, J Tribol 2009; 131: 031605-1-031605-7

[7] Ahmed SM, Hokkirigawa K, Oba, R. Fatigue failure of SUS 304 caused by vibratory cavitation erosion. Wear 1994; 177:129- 137.

[8] Ahmed SM, Hokkirigawa K, ItoY, Oba R, Matsudaira Y. Scanning electron microscopy observation on the incubation period of vibratory cavitation erosion. Wear 1991;142: 303-314.

[9] Miyazaki K, Ahmed SM, Oba R. High speed observations of the vibratory cavitation accompanying hard erosion, JSME international journal. Ser. B, Fluids and thermal engineering 1993; 36(4): 511-516.

[10] Abouel-Kasem A, Ahmed SM. Bubble structures between two walls in ultrasonic cavitation erosion. ASME, J Tribol 2012; 134(2): 02172-1-9.

[11] Abouel-Kasem A, Emara KM, Ahmed SM. Characterizing cavitation erosion particles 
by analysis of SEM images. Trib Int 2009; 42(1); 130-136.

[12] Abouel-Kasem A, Saleh B, Ahmed SM. Quantitative analysis of cavitation erosion particle morphology in dilute emulsions. ASME, J Tribol 2008; 130: 041603-1-6.

[13] Pohl M, Stella J. Quantitative CLSM roughness study on early cavitation-erosion damage. Wear 2002; 252: 501-511.

[14] Szkodo M. Relationship between microstructure of laser alloyed af C45 steel and its cavitation resistance, Proceedings of the 13th Scientific International Conference "Achievements in Mechanical and Materials Engineering" AMME'2005, GliwiceWisáa, 2005: 643-646.

[15] Abouel-Kasem A, Alturki FA, Ahmed SM. Fractal analysis of cavitation eroded surface in dilute emulsions. Trans. ASME, J Tribol 2011;133(4): 041403-1-9.

[16] Alturki FA, Abouel-Kasem A, Ahmed SM. Characteristics of cavitation erosion using image processing techniques. J Tribol 2013; 135: 041403-1-7.

[17] Lewis AS, Knowles G. Image compression using the 2-D wavelet transform, Image Processing. IEEE Trans. 1992; 1(2): 244 -250.

[18] Huang K, Aviyente S.Wavelet feature selection for image classification. IEEE Trans. Image Process 2008;17(9): 1709-1720.

[19] Mallat S. A wavelet tour of signal processing. New York :Academic; 1999

[20] Russ JC. Fractal Surfaces, New York: Plenum; 1994.

[21] Kassim AA, Zhu M, Mannan MA. Texture analysis using fractals for tool wear monitoring. IEEE ICIP 2002: III - 105- III-108.

[22] Guessasma S, Montavon G, Coddet C. On the implementation of the fractal concept to quantity thermal spray deposit surface characteristics. Surf Coat Technol 2003; 173(1): 24-38.

[23] Ribeiro LMF, Horovistiz AL, Jesui'no GAO, Hein LR, Abbade NP, Crnkovic SJ. Fractal analysis of eroded surfaces by digital image processing. Mater Lett 2002; 56:512-517.

[24] Rawers J, Tylczak J. Fractal characterization of wear-erosion surfaces. J Mater Eng Perform 1999; 8(6): 669-676.

[25] Yuan CQ, Li J, Yan XP, Peng Z. The use of the fractal description to characterize engineering surfaces and wear particles, Wear 2003; 255: 315-326.

[26] Stachowiak GW, Podsiadlo P. Characterization and classification of wear particles and surfaces. Wear 2001; 249:194-200.

[27] Podsiadlo P, Stachowiak GW. Scale-invariant analysis of wear particle morphology-i preliminary study. Tribol. Int. 33, 289-295(2000).

[28] Stachowiak GW. Numerical characterization of wear particles morphology and angularity of particles and surfaces. Tribol. Int. 31(1-3), 139-157(1998).

[29] Russ JC. Fractal analysis. Encyclopedia of Material: Science of Technology, Elsevier Science Ltd 2001: 3247-3254.

[30] Zhang, J. Detection and monitoring of wear using imaging methods. Ph.D. thesis, The Netherlands:, University of Twente, Enschede; 2006.

[31] Babadagli T, Develi K. Fractal analysis of natural and synthetic fracture surfaces of geothermal reservoir rocks. In: Proc. World Geothermal Congress 2000 Kyushu Tohoku, Japan, May 28 - June 10; 2000.

[32] Ahmed SM, Hokkirigawa K, Oba R, Kikuchi K. SEM observation of vibratory cavitation fracture-mode during the incubation period and the small roughness effect. JSME Int J 1992; 34(3): 298-303.

[33] Ahmed SM, Hokkirigawa K, Kikuchi K, Higuchi J, Oba R. SEM studies of particles produced by cavitation erosion. JSME Int. J. Ser. B 1993; 36(4): 517-523.

[34] Ahmed SM. Investigation of the temperature effects on induced impact pressure and cavitation erosion. Wear 1997; 218: 119-127.

[35] Saleh B, Abouel-Kasem A, Ezz El-Deen A, Ahmed SM. Investigation of temperature 
effects on cavitation erosion behavior based on analysis of erosion particles.Trans ASME, J Tribol 2010; 132: 031601-6.

[36] Ahmed SM, Hokkirigawa K, ItoY, Oba R, Matsudaira Y. Developing stages of ultrasonically produced cavitation erosion and corresponding surface roughness. JSME Int. J. Ser. II 1990; 33(1): 11-16.

[37] Yabuki A, Noishiki K, Komori K, Matsumura M. The surface behavior of metallic materials during the incubation period of cavitation erosion. ASTM STP 2001; 1339:357-369.

[38] Matsumura M. Erosion-corrosion: An Introduction to Flow Induced Macro-Cell Corrosion. Bentham e Books; 2012.

[39] Tao L, Song SZ, Zhang XY, Zhang Z, Lu F. Image analysis of atmospheric corrosion of field exposure high strength aluminum alloys. Appl Surf Sci 2008; 254: 6870-6874. 


\title{
"التحقيق في تقدم أضرار تآكل التكهف باستخدام

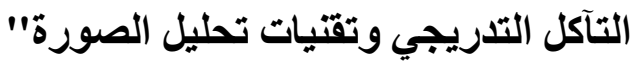

\author{
الملخص العربى التمبن
}

تم الكثف و التقييم لعملية التآكل الناتج عن التهكف للفو لاذ المقاوم للصدأ خلال فترة الاحتضسان باستخدام تقنية

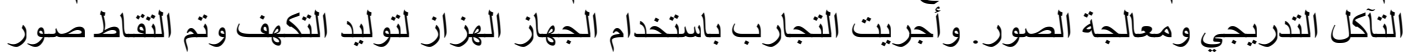

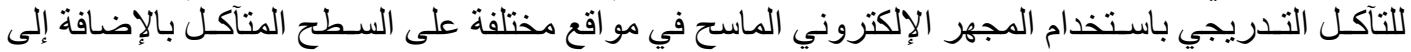

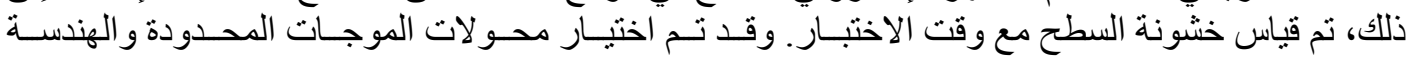

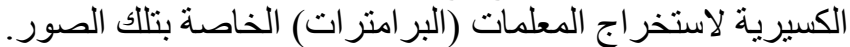

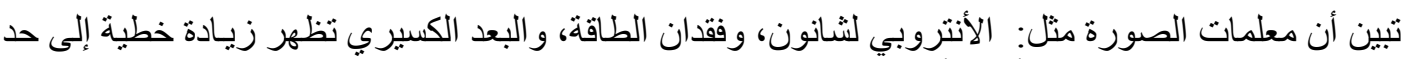

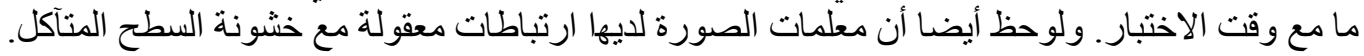

